\title{
Effective Treatment of Dystonia with Deep Brain Stimulation and Tetrabenazine in Pantothenate Kinase-Associated Neuro- degeneration: A Case Report
}

\author{
Isabel C Londoño ${ }^{1^{*}}$ (D) Luz Miriam Leiva ${ }^{1}$ (D) and Hamilton Delgado Argote ${ }^{2}$ (D) \\ ${ }^{1}$ Department of Physical Medicine and Rehabilitation, Hospital Universitario del Valle, Universidad del Valle, \\ Colombia \\ ${ }^{2}$ Faculty of Health Sciences, ICESI University, Colombia
}

*Corresponding author: Isabel Cristina Londoño, Department of Physical Medicine and Rehabilitation, Hospital Universitario del Valle, Cl. 5 \# 36-08, first floor, Cali, Colombia, Tel: 6206000, ext: 1541

\begin{abstract}
Introduction: Pantothenate kinase-associated neurodegeneration is a rare disease, difficult to diagnose and treat. It is characterized by a progressive extrapyramidal dysfunction with typical onset in the first two decades of life and by a set of clinical manifestations such as speech disturbance, focal or generalized dystonia, pigmentary retinopathy associated with mild cognitive impairment.

Clinical case: 10-year-old male patient, who until this age was managed under a diagnosis of cerebral palsy sequelae with poor response to various pharmacological regimens for the control of dystonias. After a brain MRI that showed an "Eye-of-the-Tiger" sign and a molecular test that confirmed a mutation of the PANK 2 gene, pantothenate kinase-associated neurodegeneration was diagnosed. Faced with dystonia refractory to pharmacological management, he was treated with bilateral deep brain stimulation of the globus pallidus in combination with tetrabenazine, with favorable results in functionality without significant adverse effects.

Conclusion: Pantothenate kinase-associated neurodegeneration is a pathological condition that must be taken into account in the differential diagnoses of regression in developmental milestones. The combination of tetrabenazine with deep brain stimulation is presented as an effective and safe therapeutic option for the treatment of the typical presentation of this disease.
\end{abstract}

\section{Keywords}

Neurodegeneration with brain iron accumulation, Pantothenate kinase-associated neurodegeneration, Dystonia, Globus pallidus, Eye-of-the-Tiger, Deep brain stimulation, Tetrabenazine

\section{Introduction}

Neurodegeneration with brain iron accumulation (NBIA) comprises a heterogeneous group of disorders characterized by progressive iron accumulation in the basal ganglia [1]. One of the subtypes is the pantothenate kinase-associated neurodegeneration (PKAN), a rare disease, difficult to diagnose and treat, being often recognized after several years of onset of symptoms [2]. PKAN is caused by the mutation of the PANKA2 gene that codes for the mitochondrial kinase pantothenate kinase, an essential regulatory enzyme in the biosynthesis of coenzyme $A(C O A)[2,3]$. The deficit in the production of CoA generates the accumulation of cysteine; chelator of iron at the brain level, generating the accumulation of iron in specific parts of the brain such as the basal ganglia [4]. It is a disease whose clinical manifestations can be early (in childhood or adolescence) or during early adulthood. The latter being the atypical presentation, which is slower in progression [4]. It is characterized by a progressive extrapyramidal dysfunction with typical onset in the first two decades of life and by a set of clinical manifestations such as speech disturbance, focal or generalized dystonia, pigmentary retinopathy, parkinsonian characteristics, cognitive disorders and impulsive behavior [2]. If this clinical picture is associated with brain magnetic resonance imaging (MRI) showing the "Eye-of-the-Tiger" sign; it increases the diagnostic probability, since this is considered a classic finding [3]. PKAN

Citation: Londoño I, Leiva LM, Delgado H (2021) Effective Treatment of Dystonia with Deep Brain Stimulation and Tetrabenazine in Pantothenate Kinase-Associated Neurodegeneration: A Case Report. Int J Brain Disord Treat 6:037. doi.org/10.23937/2469-5866/1410037

Accepted: May 29, 2021: Published: May 31, 2021

Copyright: (C) 2021 Londoño l, et al. This is an open-access article distributed under the terms of the Creative Commons Attribution License, which permits unrestricted use, distribution, and reproduction in any medium, provided the original author and source are credited. 


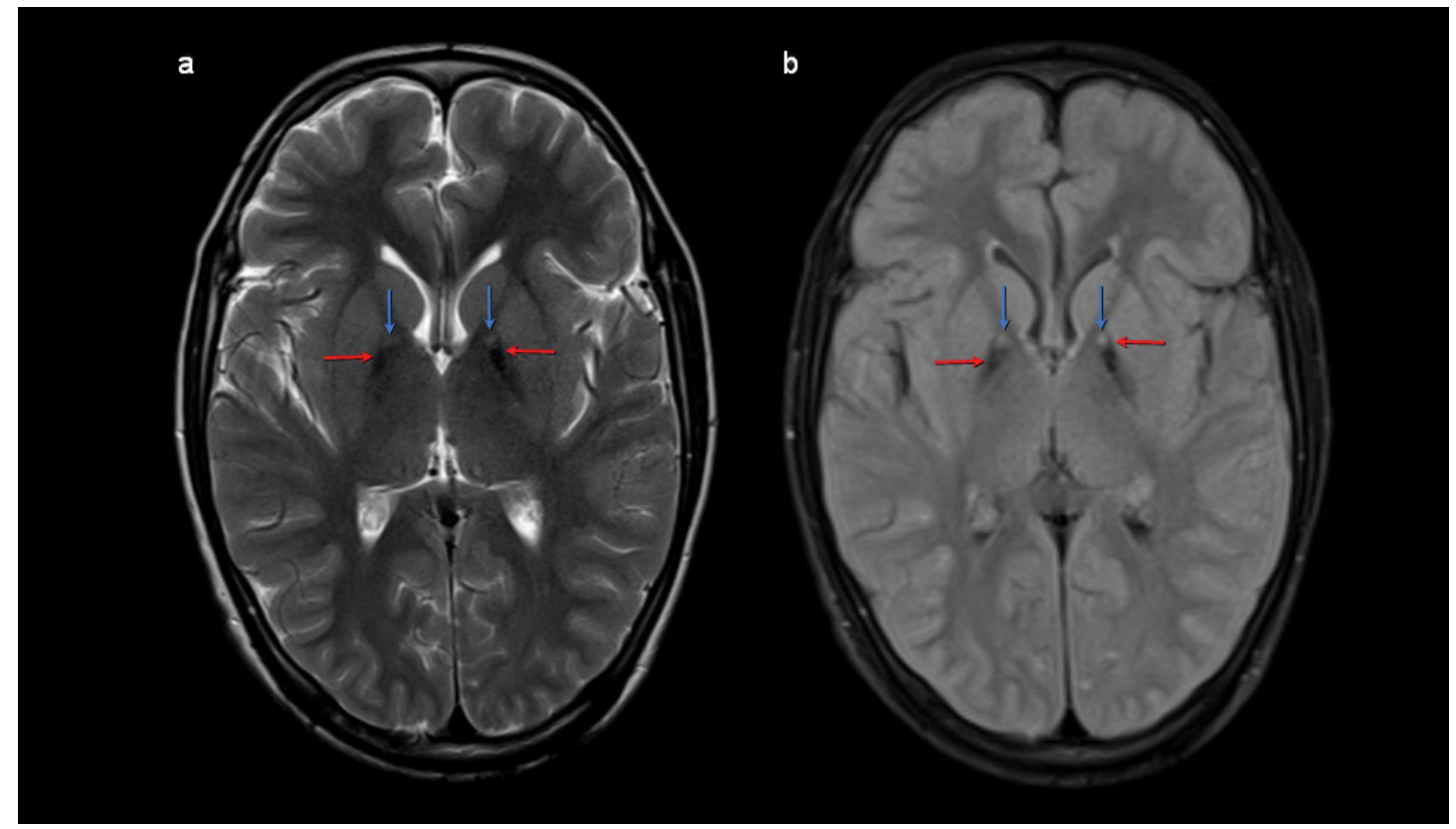

Figure 1: Axial brain magnetic resonance imaging (MRI) weighted in T2 (a) and T2 FLAIR (b) at the level of the basal ganglia showing hypointensities in the medial margin of both globus pallidus (red arrow) consistent with iron deposits, with a punctiform hyperintense image (blue arrows) corresponding to gliosis/spongiosis foci.

causes a generalized, progressive dystonia, normally refractory to pharmacological management, which is why in recent years, deep brain stimulation has been shown to be effective in controlling symptoms and improving the quality of life of patients $[2,5,6]$.

PKAN also named Hallervorder-Spatz syndrome; it has been suggested to abandon this eponymous considering the ethical failures in human research of these doctors in the Second World War [7].

The child up to 10 years of age was managed under the diagnosis of cerebral plasy sequelae, with poor response to various pharmacological regimens for the control of dystonias. After studies, the diagnosis of PKAN was clarified and he was treated with bilateral deep brain stimulation of the globus pallidus in combination with tetrabenazine, with favorable results.

\section{Clinical Case}

10-year-old male, resident in Colombia. Product of first pregnancy without complications, of a 26 -year-old mother and 32-year-old father, not consanguineous. No significant family history. He was born at term due to delivery, with normal weight, height and adaptation at birth, he did not require resuscitation or stay in the ICU. Within the developmental milestones, he had cephalic control at 5 months, seated posture at 7 months, standing and independent gait at 2 years with first words at 5 years. He was referred to the Child Rehabilitation Physiatry consultation for the first time at the age of 9 with a diagnosis of delay in psychomotor development, secondary to motor impairment of central origin, to start a rehabilitation plan. During the questioning, the mother mentioned falls backwards, described as episodes of ri- gidity without any protective reflex, difficulty communicating and chewing food. On physical examination, expressionless facial features are striking, dysarthria due to spasticity in the orofacial muscles, dystonic movement of the right upper limb, toe gait, patellar hyperreflexia, Achilles clonus, increased tone in bilateral gastrocnemius with preserved upper brain functions. Studies were requested and a rehabilitation plan with physical, occupational and speech therapy started. Six months later, dystonia became generalized with marked deterioration of function until prostration, with difficulty feeding, marked weight loss, and poor response to stimuli. The emergency department was consulted due to difficulty controlling dystonic movements and pain, hospitalization was indicated. Wilson's disease was ruled out and management with multiple therapeutic alternatives such as baclofen, carbidopa and levodopa, benzodiazepines, haloperidol, and tizanidine stared, obtaining little response. Given the refractoriness, it was considered to start with tetrabenazine (TBZ) $25 \mathrm{mg}$ tablet, starting with $12.5 \mathrm{mg} /$ day and increasing $12.5 \mathrm{mg}$ every 5 days, reaching doses of $100 \mathrm{mg} /$ day. During hospitalization, T2-weighted brain MRI was performed, which showed bilateral symmetric hypointensity in the globus pallidus with central hyperintensity, giving an Eye-of-the-Tiger sign (Figure 1) without other alteration, suggesting neurodegeneration associated with pantothenate kinase. A genetic study was requested, exomic sequencing, which confirmed the mutation of the PANK 2 gene (Table 1).

He was evaluated in an interdisciplinary board of abnormal movements, made up of a neurosurgeon, radiologist, pediatric neurologist and physiatrist, deciding the relevance of implantation of a deep brain stimulator. To the 10 -years-old, implanted bilateral DBS 


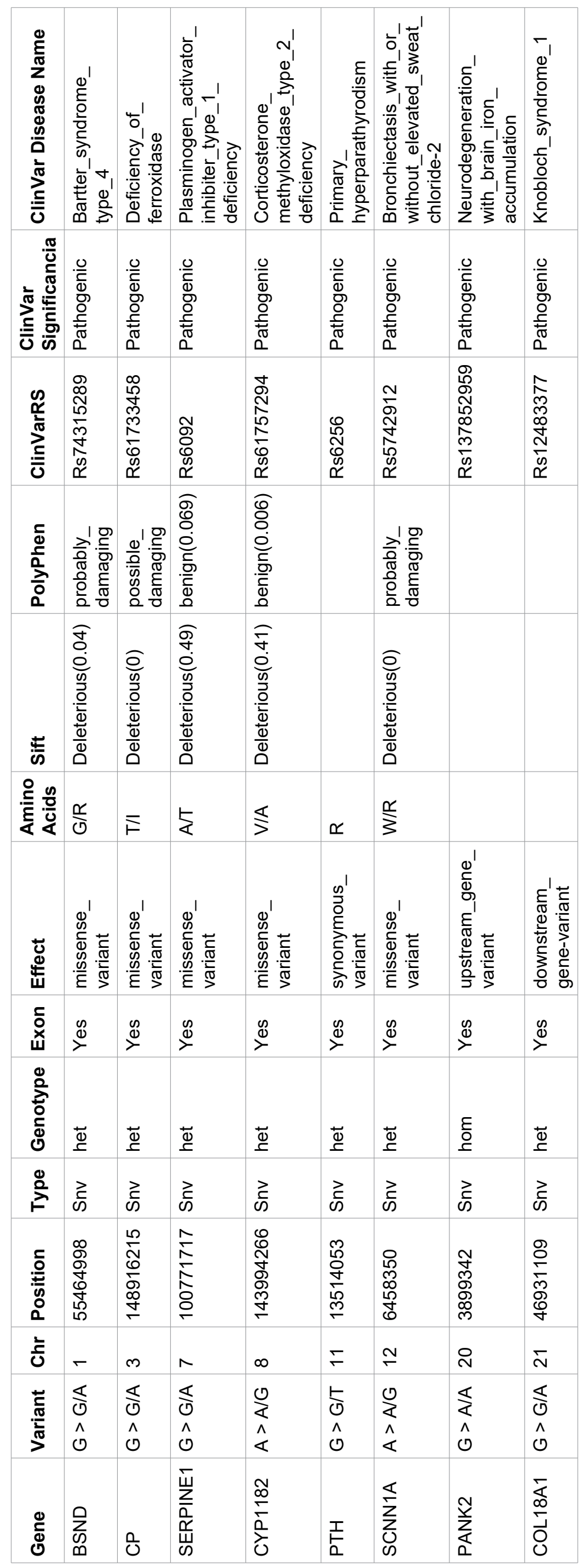




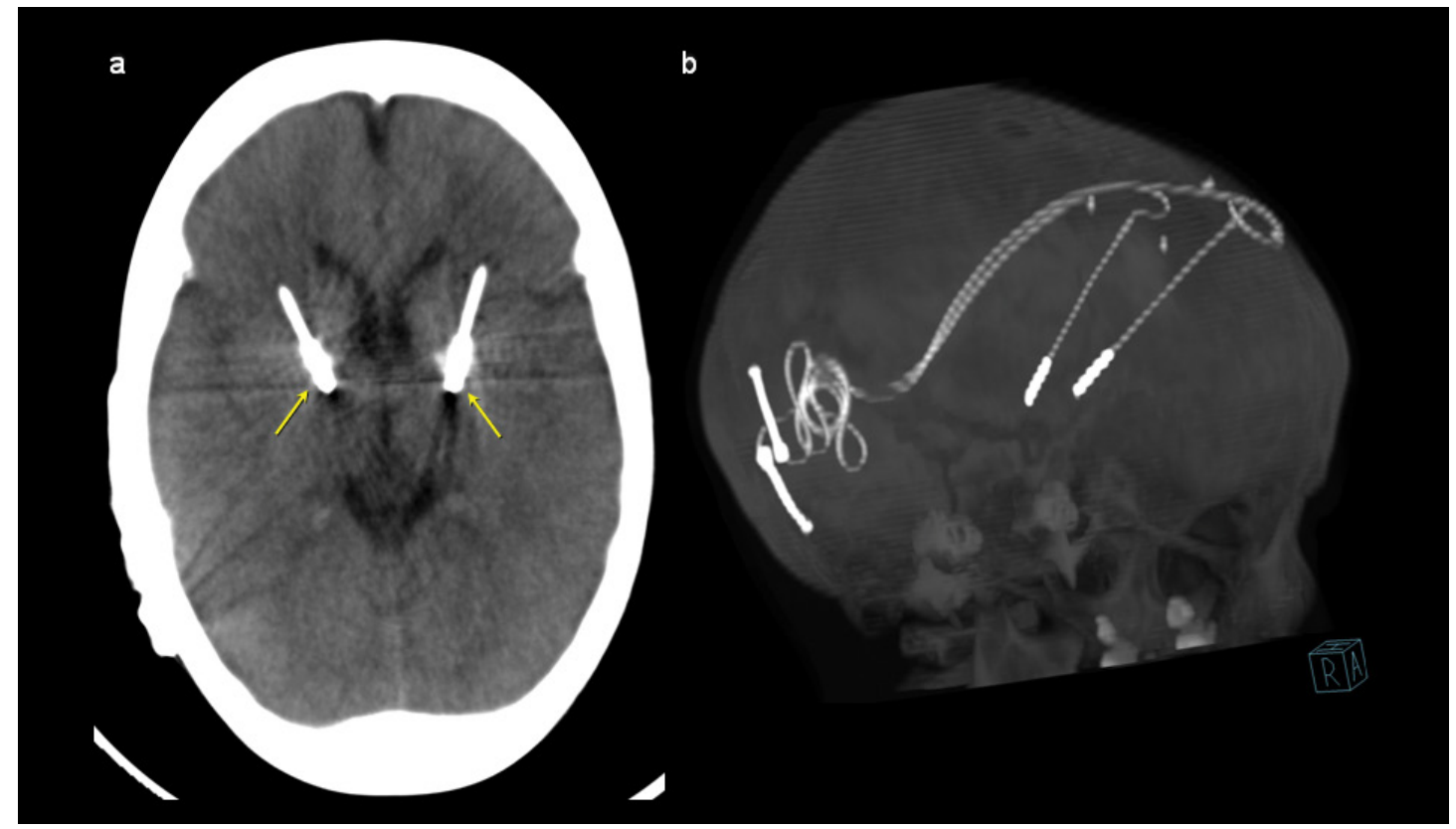

Figure 2: Maximum intensity projection (MIP) axial computed tomography images (a) and 3D MIP reconstruction (b) showing the location of the neurostimulator electrodes, with their distal end located in the anteromedial margin of both globus pallidus (yellow arrows).

electrodes into the globus pallidus internus, the VerciseTM stimulator, model DB-6412-EU-C, Boston Scientific, the DBS system was programmed to a monopolar stimulation a frequency of $85 \mathrm{~Hz}$, a pulse width of 100 $\mu \mathrm{s}$, and an amplitude of $2.6 \mathrm{~mA}$. One month after the procedure, the patient showed evident improvement, after being in bed, he starts rolling, achieves a quadruped posture, performs oral opening, tolerating feeding with an acceptable chewing pattern, improves dystonic movement in hyperextension of the right elbow allowing to recover hand mouth pattern and use of spoon. Two months after the implantation of the stimulator, weight gain, improved mood, interaction with the environment and social participation were observed. The patient continued in medical follow-up by physiatry periodically for 4 years, observing control of the dystonia; the dose of tetrabenazine was adjusted to $200 \mathrm{mg} /$ day. So far, he has not presented symptoms of dementia or depression, sometimes irritability at stressful moments like mealtime (Figure 2).

\section{Discussion}

Not all early neurodevelopmental disorders are due to cerebral palsy, and the spectrum of NBIA should be considered in differential diagnoses. A detailed clinical history is important for the diagnosis, evaluating neurodevelopment, performing a complete neurological and musculoskeletal physical examination, in addition to identifying the abnormal movements that accompany the picture $[2,4]$.

Ophthalmology assessment is important to detect retinitis pigmentosa, which is common in these patients. Brain MRI is required since the "Eye-of-the-Tiger" sign, consisting of a hyperintense focus in the an- teromedial portion of the globus pallidus surrounded by a hypointense area that covers the posterior portions of this nucleus, when evaluating T2-weighted sequences, is a classic and sensitive finding that allows differentiating PKAN from other varieties of NBIA [3]. The absolute correlation of this sign and PKAN has been questioned by other authors, suggesting that its appearance may change over time with an increase in iron accumulation, the latter being responsible for the low signal in the periphery of the "eye" [3]. At present we have available genetic tests that confirm the mutation of the PKAN 2 gene by means of exomic sequencing $[2,4]$.

PKAN does not have a specific treatment or disease-modifying treatment [2]. It requires a multidisciplinary approach, with the participation of neurologist, geneticist, ophthalmologist, radiologist, neurosurgeon, physiatrist and rehabilitation group; physiotherapist, occupational therapist and speech therapist. Dystonia is one of the major conditions and the most disabling, it can be managed with carbidopa/levodopa, benzodiazepine and baclofen, sometimes reaching maximum doses without response and presenting adverse effects of the medication $[2,8]$. In our patient, due to refractoriness to pharmacological management, TBZ was considered, a second-line drug in PKAN dystonia [2]. TBZ is an inhibitor of the reuptake of monoamines (serotonin, dopamine, norepinephrine, histamine) in the presynaptic vesicles of neurons. It acts through type 2 transporters of monoamine vesicles, reducing their availability in nerve terminals $[2,8]$. It has been used for the treatment of various hyperkinetic movement disorders with evidence of effectiveness in Huntington's disease, tardive dyskinesia, tics and Gilles de la Tourette syndrome $[2,9]$. Studies reported on the effectiveness of TBZ in im- 
proving various types of dystonia in pediatric and adult patients [8]. TBZ has been used in doses ranging from $25-200 \mathrm{mg} /$ day, although the most common range is 50 $75 \mathrm{mg} /$ day. The dose adjustment should be done slowly, starting with doses of $12.5 \mathrm{mg} /$ day at night and making increments of $12.5 \mathrm{mg}$ every 4-7 days, until reaching the doses considered effective or at which relevant adverse effects appear [2,9]. In the case described, a gradual increase was made to $200 \mathrm{mg} /$ day. The daily dose should be divided into three doses, although in some cases the administration in two daily doses is satisfactory in terms of efficacy and tolerability $[2,9]$.

Previously, when the pharmacological treatment did not work, the procedure of choice was the ablation of the globus pallidus (pallidotomy) but it has fallen into disuse because it is irreversible and because of the adverse effects it generates, including cognitive impairment. In 1977, a new concept was introduced, electrical stimulation of the globus pallidus, which is performed by means of surgically inserted electrodes, to inhibit neuronal activity activated pathologically $[1,5,6]$; with notable benefit in language, speech and swallowing, improvement in axial dystonia, pain and quality of life, as we saw in our patient. Complications include electrode displacement, infection, and source depletion [5]. Cognition and mood are not affected, unlike the ablative, in this case the mother reports that he presented improved mood and participation in peer play. Castelnau, et al. analyzed a group of 6 children with PKAN over a period of twenty months, finding that deep brain stimulation is a durable solution for the treatment of severe generalized dystonia in patients with PKAN; with improvement in pain, dystonia and functionality with few adverse effects [10]. However, Krause, et al, reported a case the a 13-year-old male with the typical clinical features of PKAN, they implanted him a DBS, followed up for 5 years and document deterioration of motor function; not by a decrease in DBS efficacy but rather a change in the clinical presentation of PKAN due to disease progression [11]. Are necessary more studies to determine possible prognostic factors and long-term benefit for pallidal DBS in PKAN.

It has been observed that the degree of improvement in dystonia is directly proportional to the severity of the preoperative dystonia, and that prolonged disease duration and skeletal deformities are associated with unfavorable postoperative outcomes. For this reason, deep brain stimulation using an implantable neurostimulator must be considered in a timely manner, to obtain greater benefits and improve the lives of these patients $[1,5,6]$. PKAN is a pathology that is considered as a differential diagnosis in regression in developmental milestones. This case exposes the combination of tetrabenazine with deep brain stimulation as an effective and safe therapeutic option for the treatment of the typical presentation of this pathology. However, there is little information regarding the combination of deep brain stimulation with tetrabenazine for the treatment of generalized dystonia, and more studies are required.

\section{Conflicts of Interest}

The authors declare that they have no conflicts of interest for the writing of this article.

\section{Funding}

We have not received funding.

\section{References}

1. Sathe KP, Hegde AU, Doshi PK (2013) Deep brain stimulation improves quality of life in pantothenate kinase-associated neurodegeneration. J Pediatr Neurosci 8: 46-48.

2. Hogarth $P$, Kurian MA, Gregory A, Csányi B, Zagustin T, et al. (2017) Consensus clinical management guideline for pantothenate kinase-associated neurodegeneration (PKAN). Mol Genet Metab 120: 278-287.

3. Vilchez-abreu C, Roa-sanchez $P$, Fermin-delgado $R$, Speckter H, Perez-then E, et al. (2013) The "tiger's eye" sign on MRI: Age-related changes. An Radiol México 12: 189-196.

4. Razmeh S, Habibi AH, Orooji M, Alizadeh E, Moradiankokhdan K, et al. (2018) Pantothenate kinase-associated neurodegeneration: clinical aspects, diangnosis and treatments. Neurol Int 10: 32-34.

5. Tsering D, Tochen L, Lavenstein B, Reddy SK, Granader $Y$, et al. (2017) Considerations in deep brain stimulation (DBS) for pediatric secondary dystonia. Child's Nerv Syst 33: 631-637.

6. Garcia-Ruiz PJ, Ayerbe J, Vela Desojo L, Feliz CE, del Val Fernandez J (2015) Deep brain stimulation for pantothenate kinase-associated neurodegeneration. Case Rep Neurol Med 2015: 245735

7. Zeidman LA, Pandey DK (2012) Declining use of the Hallervorden-Spatz disease eponym in the last two decades. J Child Neurol 27: 1310-1315.

8. Luc QN, Querubin J (2017) Clinical management of dystonia in childhood. Pediatr Drugs 19: 447-461.

9. Chen JJ, Ondo WG, Dashtipour K, Swope DM (2012) Tetrabenazine for the Treatment of Hyperkinetic Movement Disorders: A Review of the Literature. Clin Ther 34: 1487-504.

10. Castelnau P, Cif L, Valente EM, Vayssiere N, Hemm S, et al. (2005) Pallidal stimulation improves pantothenate kinase - associated neurodegeneration. Ann Neurol 57: 738-741.

11. Krause M, Fogel W, Tronnier V, Pohle S, Hörtnagel K, et al. (2006) Long-term benefit to pallidal deep brain stimulation in a case of dystonia secondary to pantothenate kinase-associated neurodegeneration. Mov Disord 21: 2255-2257. 